\title{
Set-Valued Weighted Value at Risk and Its Computation
}

\author{
Yichuan Dong 1,2, Yijun $\mathrm{Hu}^{1,2}$ and Yu Feng 1,2* \\ ${ }^{1}$ School of Mathematics and Statistics, Wuhan University, Wuhan, China, ${ }^{2}$ National Supercomputing Center in Shenzhen, \\ Shenzhen, China
}

In this paper, we propose a new class of set-valued coherent risk measures called the set-valued weighted value at risk. Firstly, the "regulator" version is independent of other market scenarios. The second version, which is called the market extension, is related to different market scenarios. The proofs of the properties of both versions are given, and equivalent representations are provided that enable us to compute the values of both versions of set-valued weighted value at risk. Finally, we offer examples to illustrate various features of the theoretical constructions of the set-valued weighted value at risk.

\section{OPEN ACCESS}

Edited by:

Muhammad Javaid,

University of Management and

Technology, Lahore, Pakistan

Reviewed by:

Su Guicong,

Jagiellonian University, Poland

Chunyan Li,

Chongqing University of Science and

Technology, China

Liu Xi Xian,

University of International Business and Economics, China

*Correspondence:

Yu Feng

fengyuffyy@whu.edu.cn

Specialty section:

This article was submitted to

Mathematical and Statistical Physics,

a section of the journal

Frontiers in Physics

Received: 29 March 2020

Accepted: 30 April 2020

Published: 19 June 2020

Citation:

Dong Y, Hu Y and Feng Y (2020)

Set-Valued Weighted Value at Risk

and Its Computation.

Front. Phys. 8:190.

doi: 10.3389/fphy.2020.00190
Mathematics Subject Classification (2010): 91B30 91B3291B70.

Keywords: set-valued coherent risk measure, set-valued average value at risk, set-valued weighted value at risk, representation, market extension

\section{INTRODUCTION}

Weighted value at risk for one-dimension random variables may be one of the most popular coherent risk measures (see [1]). Artzner et al. [2] initially introduced the first coherent risk measure by proposing four axioms. Cherny [1] showed that weighted value at risk possesses some desirable properties that are not shared by Expected Shortfall. For further details on Expected Shortfall, we refer the reader to Föllmer and Schied [3]. Weighted value at risk first appeared in Kusuoka [4]. Acerbi [5, 6] called it the spectral risk measure.

Jouini et al. [7] demonstrated that a set of set-valued risk measures are suitable for evaluating multivariate risks in market models with transaction costs/bid-ask spreads. Additional set-valued risk measures have since been introduced and studied (see [8-13], and the references therein).

Hamel et al. [11] introduced set-valued average value at risk, and reasons for using setvalued functions as risk measures have been further addressed from both financial and mathematical perspectives (see [14-17]), and the reference there in).

In this paper, we will extend the traditional weighted value at risk to a set-valued version for multivariate random variables. Therefore, we demonstrate their core properties and provide an alternative representation for computing their values. The first version is called "regulator weighted value at risk" since it does not take trading opportunities into account. The second version is called "the market extension" since it relates to a specific market scenario. These two versions are set-valued coherent risk measures. Then, we derive a benchmark when introducing set-valued weighted value at risk that can reflect the risk tolerance of the trader/regulator; see Remark 2.2 below. Finally, we offer examples to illustrate various features of the theoretical constructions of the set-valued weighted value at risk.

The remainder of this article is organized as follows. Section 2 introduces a primal and an equivalent representation of set-valued weighted value at risk, including the "regulator" and "the market extension" cases. The essential properties of both cases are then proven. In section 3, 
examples are given to illustrate the theoretical construction of the set-valued weighted value at risk.

\section{SET-VALUED WEIGHTED VALUE AT RISK}

\subsection{The Regulator Case}

Let $(\Omega, \mathscr{F}, P)$ be a probability space and $d \geq 1$ be a positive integer. A multivariate random variable is an $\mathscr{F}$-measurable function $\mathbf{X}: \Omega \rightarrow \mathbf{R}^{d}$ for $d \geq 2$. Here, $d=1$ represents a one-dimension random variate. Denote by $L_{d}^{0}:=L_{d}^{0}(\Omega, \mathscr{F}, P)$ the linear space of the equivalence classes (with respect to the probability $P$ ) of $\mathbf{R}^{d}$-valued random variables. An element $\mathbf{X} \in L_{d}^{0}$ has components $X_{1}, \cdots, X_{d}$ in $L^{0}:=L_{1}^{0}$. Denote by $\left(L_{d}^{0}\right)_{+}$the set of $\mathbf{R}^{d}$-valued random variables with $P$ almost surely nonnegative components and by $L_{d}^{1}:=L_{d}^{1}(\Omega, \mathscr{F}, P)$ the linear space of all $\mathbf{X}=\left(X_{1}, \cdots, X_{d}\right) \in L_{d}^{0}$ with $\int_{\Omega} X_{i} d P<+\infty, 1 \leq$ $i \leq d$. We also define $E[\mathbf{X}]=\left(E X_{1}, \cdots, E X_{d}\right)^{T}$ for $\mathbf{X} \in L_{d}^{1}$, the transpose of row vector $\left(E X_{1}, \cdots, E X_{d}\right)$. Define $\left(L_{d}^{1}\right)_{+}=$ $L_{d}^{1} \cap\left(L_{d}^{0}\right)_{+}$. If $d=1$, we write $L^{0}, L_{+}^{0}$ and $L_{+}^{1}$ for $L_{1}^{0},\left(L_{d}^{0}\right)_{+}$ and $\left(L_{d}^{1}\right)_{+}$, respectively. For $\alpha \in \mathbf{R}^{d}$, the symbol $\operatorname{diag}(\alpha)$ denotes the $d \times d$ matrix with the components of the vector $\alpha$ as entries on its main diagonal and zero entries elsewhere. $x^{+}$ stands for $\max (x, 0)$ for $x \in \mathbf{R}$ [see [18-21]] and the reference therein).

The next definition offers an essential representation for setvalued weighted value at risk, which is an extension of the scalar case given by Cherny [1] to the set-valued case. It involves a linear subspace $\mathbf{M} \subseteq \mathbf{R}^{d}$, called the space of eligible assets, which we adopt from Hamel et al. [11]. We will also employ a benchmark level, which is one of the novelties of this article; see Remark 2.2 below. A natural choice for $\mathbf{M}$ is $\mathbf{M}=\mathbf{R}^{m} \times$ $\{0\}^{d-m}, 1 \leq m \leq d$, i.e., the first $m$ of $d$ assets are eligible as deposits (see $[\overline{7}, 11,22])$. We denote $\mathbf{M}_{+}=\mathbf{M} \cap \mathbf{R}_{+}^{d}$, where $\mathbf{R}_{+}^{d}$ stands for the class of elements in $\mathbf{R}^{d}$ with nonnegative components. We assume that $\mathbf{M}_{+}$is non-trivial, i.e., $\mathbf{M}_{+} \neq\{\mathbf{0}\}$.

Generally speaking, a scalar multivariate risk measure is any mapping from $L_{d}^{0}$ to $\mathbf{R}$. A set-valued risk measure is any mapping $\rho$ from $L_{d}^{0}$ to a class of subsets of $\mathbf{R}^{d} \cdot \rho(\mathbf{X})$ is interpreted as a set of acceptable margins of portfolio $\mathbf{X}$ (see [23-27]) and the reference therein).

Definition 2.1 Let $\theta \in(0,1)$ and $\mu:=\left(\mu_{1}, \cdots, \mu_{d}\right)$ be a probability on $[\theta, 1]^{d}$. For $\mathbf{X} \in L_{d}^{0}$, the set-valued weighted value at risk at $\mathbf{X}$ with respect to $\mu$ is defined as

$$
\begin{aligned}
& W \operatorname{VaR}_{\mu}(\mathbf{X}):=\left\{\int_{[\theta, 1]^{d}} \operatorname{diag}(\alpha)^{-1} E[\mathbf{Z}] \mu(d \alpha)-z ;\right. \\
&\left.\mathbf{Z} \in\left(L_{d}^{1}\right)_{+}, \mathbf{X}+\mathbf{Z}-z \in\left(L_{d}^{0}\right)_{+}, z \in \mathbf{R}^{d}\right\} \cap \mathbf{M},
\end{aligned}
$$

where $\quad \int_{[\theta, 1]^{d}} \operatorname{diag}(\alpha)^{-1} E[\mathbf{Z}] \mu(d \alpha) \quad-\quad z \quad:=$ $\left[\int_{[\theta, 1]} \frac{1}{\alpha_{i}} E\left[Z_{i}\right] \mu_{i}\left(d \alpha_{i}\right)-z_{i}\right]_{i=1}^{d}:=\left(\int_{[\theta, 1]} \frac{1}{\alpha_{1}} E\left[Z_{1}\right] \mu_{1}\left(d \alpha_{1}\right)-\right.$ $\left.z_{1}, \cdots, \int_{[\theta, 1]} \frac{1}{\alpha_{1}} E\left[Z_{d}\right] \mu_{d}\left(d \alpha_{d}\right)-z_{d}\right)^{T}$ for $\mathbf{Z}=\left(Z_{1}, \cdots, Z_{d}\right) \in$ $\left(L_{d}^{1}\right)_{+}$and $z=\left(z_{1}, \cdots, z_{d}\right) \in \mathbf{R}^{d}$.
Remark 2.1 If $\mu$ is a Dirac measure at some $\alpha \in(0,1]^{d}$, that is, $\mu(\{\alpha\})=1$, then Definition 2.1 reverts to the definition of the set-valued regulator average value at risk of Hamel et al. [11] (Definition 2.1) because the benchmark level $\theta$ can be small enough. Moreover, in Example 3.2 below, we show that the $W V a R_{\mu}$ is better suited to the change in the market than the regulator average value at risk of Hamel et al. [11].

Remark 2.2 The financial interpretation of the benchmark level $\theta$ is as follows. Initially, it stems from the confidence level $1-\alpha$ of value at risk. Given a confidence level $1-\alpha \in(0,1)$, the value at risk at $X \in L^{0}$ is defined as $\operatorname{VaR}_{1-\alpha}(X):=\inf \{t \in \mathbf{R} ; P(X>t) \leq \alpha\}$. From a practical perspective, in reality, the parameter $1-\alpha$ can be very close to but cannot be 1 . Thus, $\alpha$ can be very close to but cannot be zero, which motivates the introduction of the benchmark level $\theta$, which reflects the risk tolerance of the investor/regulator in terms of probability. See Basel Committee [28-31] for the reasonability of the benchmark level. Therefore, the benchmark level $\theta$ can be very close to zero but cannot be exactly zero. Examples 3.1 and 3.2 below take this perspective into account.

Remark 2.3 In definition 2.1, the intersection with $\mathbf{M}$ has the following interpretation. To cancel the risk of portfolio $\mathbf{X}$, we would like to obtain a set of all margins when measuring the risk of portfolio $\mathbf{X}$. Intersecting with the set $\mathbf{M}, W V a R_{\mu}(\mathbf{X})$ shows both the valid margins and the aggregated margins, which aggregates the valid margins from the $d$-dimension to the $m$ dimension. The other $(d-m)$-dimension of $W V a R_{\mu}(\mathbf{X})$ should be zero. Aggregating the margin has plenty of financial explanations. For example, each element of the vector represents the amounts in a specific currency. Suppose that $m$ different currencies should be taken into consideration. For the regulator, there is no need to ask for a $d$-dimensional margin. They could aggregate $d$ elements of the margin into $m$ elements that represent $m$ different currencies. When considering the margin needed by a company with different departments, this idea is also reasonable. The decision-maker of a company may simply want to figure out the sum of the margins of different departments. More details can be found in Jouini et al. [7].

The next proposition provides another equivalent representation of $W V a R_{\mu}$ under the condition $\mathbf{M}=\mathbf{R}^{m} \times\{0\}^{d-m}$, which is easier to compute than (2.1).

Proposition 2.1 Let $\mathbf{M}=\mathbf{R}^{m} \times\{0\}^{d-m}$ (hence $\mathbf{M}_{+}=\mathbf{R}_{+}^{m} \times$ $\left.\{0\}^{d-m}\right)$. The set-valued weighted value at risk takes the following equivalent representation:

$$
\begin{gathered}
\operatorname{WVaR}_{\mu}(\mathbf{X})=\left(\left[\inf _{z_{i} \in \mathbf{R}}\left\{\int_{[\theta, 1]} \frac{1}{\alpha_{i}} E\left[\left(-X_{i}+z_{i}\right)^{+}\right] \mu_{i}\left(d \alpha_{i}\right)-z_{i}\right\}\right]_{i=1}^{m}\right. \\
\left.+\mathbf{R}_{+}^{m}\right) \times\{0\}^{d-m}
\end{gathered}
$$

for $\mathbf{X}=\left(X_{1}, \cdots, X_{d}\right) \in L_{d}^{0}$.

Proof Considering a component of the portfolio, we know that the two conditions $Z_{i} \in L_{+}^{1}$ and $X_{i}+Z_{i}-z_{i} \in L_{+}^{0}$ are 
equivalent to $Z_{i} \geq\left(-X_{i}+z_{i}\right)^{+}$for $1 \leq i \leq d$. Therefore, $\left\{\int_{[\theta, 1]} \frac{1}{\alpha_{i}} E\left[Z_{i}\right] \mu_{i}\left(d \alpha_{i}\right)-z_{i} ; Z_{i} \in L_{+}^{1}, X_{i}+Z_{i}-z_{i} \in L_{+}^{0}, z_{i} \in \mathbf{R}\right\}$ is equal to $\inf _{z_{i} \in \mathbf{R}}\left\{\int_{[\theta, 1]} \frac{1}{\alpha_{i}} E\left[\left(-X_{i}+z_{i}\right)^{+}\right] \mu_{i}\left(d \alpha_{i}\right)-z_{i}\right\}+\mathbf{R}_{+}$. After intersecting with the set $\mathbf{M}$, we have that

$$
\begin{gathered}
\operatorname{WVaR}_{\mu}(\mathbf{X})=\left(\left[\inf _{z_{i} \in \mathbf{R}}\left\{\int_{[\theta, 1]} \frac{1}{\alpha_{i}} E\left[\left(-X_{i}+z_{i}\right)^{+}\right] \mu_{i}\left(d \alpha_{i}\right)-z_{i}\right\}\right]_{i=1}^{m}\right. \\
\left.+\mathbf{R}_{+}^{m}\right) \times\{0\}^{d-m} .
\end{gathered}
$$

Proposition 2.1 is proved.

The next proposition will show that when $\mathbf{M}=\mathbf{R}^{m} \times\{0\}^{d-m}$, the set-valued weighted value at risk is exactly a set-valued coherent risk measure in the sense of Jouini et al. [7].

Proposition 2.2 Let $\mathbf{M}=\mathbf{R}^{m} \times\{0\}^{d-m}$. Then, the function $\mathbf{X} \longrightarrow W \operatorname{VaR}_{\mu}(\mathbf{X})$ meets the listed properties:

(a) Positive homogeneity: for any $\mathbf{X} \in L_{d}^{0}$ and any $s>0$, $W \operatorname{VaR}_{\mu}(s \mathbf{X})=s W \operatorname{VaR}_{\mu}(\mathbf{X})$.

(b) Subadditivity: for any $\mathbf{X}^{1}, \mathbf{X}^{2} \in L_{d}^{0}, W \operatorname{VaR}_{\mu}\left(\mathbf{X}^{1}+\mathbf{X}^{2}\right) \supseteq$ $W \operatorname{VaR}_{\mu}\left(\mathbf{X}^{1}\right)+W \operatorname{VaR}_{\mu}\left(\mathbf{X}^{2}\right)$.

(c) M-translation invariance: for any $\mathbf{X} \in L_{d}^{0}$ and any $u \in \mathbf{R}^{m}$, $W \operatorname{VaR}_{\mu}(\mathbf{X}+\bar{u})=W \operatorname{VaR}_{\mu}(\mathbf{X})-\bar{u}$, where $\bar{u}=u \times\{0\}^{d-m}$.

(d) Monotonicity with respect to $\left(L_{d}^{0}\right)_{+}$: for any $\mathbf{X}^{1}, \mathbf{X}^{2} \in\left(L_{d}^{0}\right)_{+}$ with $\mathbf{X}^{2} \geq \mathbf{X}^{1}$, which means that $\mathbf{X}^{2}-\mathbf{X}^{1} \in\left(L_{d}^{0}\right)_{+}$, we have $W \operatorname{VaR}_{\mu}\left(\mathbf{X}^{2}\right) \supseteq W \operatorname{VaR}_{\mu}\left(\mathbf{X}^{1}\right)$.

(e) It satisfies that $W \operatorname{VaR}_{\mu}(\mathbf{X})+\mathbf{M}_{+}=W \operatorname{VaR}_{\mu}(\mathbf{X})$ for $\mathbf{X} \in L_{d}^{0}$. Particularly, $W \mathrm{VaR}_{\mu}(\mathbf{0})$ is a convex cone.

Proof (a) For $\mathbf{X}=\left(X_{1}, \cdots, X_{d}\right) \in L_{d}^{0}$ and $s>0$,

$W \operatorname{VaR}_{\mu}(s \mathbf{X})=$

$$
\begin{gathered}
\left(\left[\inf _{z_{i} \in \mathbf{R}}\left(-z_{i}+\int_{[\theta, 1]} \frac{1}{\alpha_{i}} E\left[\left(z_{i}-s X_{i}\right)^{+}\right] \mu_{i}\left(d \alpha_{i}\right)\right)\right]_{i=1}^{m}\right. \\
\left.\quad+\mathbf{R}_{+}^{m}\right) \times\{0\}^{d-m} \\
=\left(\left[\inf _{z_{i} \in \mathbf{R}}\left(-z_{i}+s \int_{[\theta, 1]} \frac{1}{\alpha_{i}} E\left[\left(\frac{z_{i}}{s}-X_{i}\right)^{+}\right] \mu_{i}\left(d \alpha_{i}\right)\right)\right]_{i=1}^{m}\right. \\
\left.\quad+\mathbf{R}_{+}^{m}\right) \times\{0\}^{d-m} \\
=\left(\left[s \inf _{z_{i} \in \mathbf{R}}\left(-\frac{z_{i}}{s}+\int_{[\theta, 1]} \frac{1}{\alpha_{i}} E\left[\left(\frac{z_{i}}{s}-X_{i}\right)^{+}\right] \mu_{i}\left(d \alpha_{i}\right)\right)\right]_{i=1}^{m}\right. \\
\left.\quad+\mathbf{R}_{+}^{m}\right) \times\{0\}^{d-m} \\
=\left(\left[s \inf _{\frac{z_{i}}{s} \in \mathbf{R}}\left(-\frac{z_{i}}{s}+\int_{[\theta, 1]} \frac{1}{\alpha_{i}} E\left[\left(\frac{z_{i}}{s}-X_{i}\right)^{+}\right] \mu_{i}\left(d \alpha_{i}\right)\right)\right]_{i=1}^{m}\right. \\
\left.\quad+\mathbf{R}_{+}^{m}\right) \times\{0\}^{d-m}
\end{gathered}
$$

$=s W V_{a} R_{\mu}(\mathbf{X})$. (b) For $\mathbf{X}^{1}=\left(X_{1}^{1}, \cdots, X_{d}^{1}\right), \mathbf{X}^{2}=\left(X_{1}^{2}, \cdots, X_{d}^{2}\right) \in L_{d}^{0}$,

$$
\begin{aligned}
& W \operatorname{VaR}_{\mu}\left(\mathbf{X}^{1}+\mathbf{X}^{2}\right)= \\
& \left(\left[\inf _{z_{i} \in \mathbf{R}}\left(-z_{i}+\int_{[\theta, 1]} \frac{1}{\alpha_{i}} E\left[\left(z_{i}-X_{i}^{1}-X_{i}^{2}\right)^{+}\right] \mu_{i}\left(d \alpha_{i}\right)\right)\right]_{i=1}^{m}\right. \\
& \left.+\mathbf{R}_{+}^{m}\right) \times\{0\}^{d-m} \\
& \supseteq\left(\left[\operatorname { i n f } _ { z _ { i } ^ { 1 } + z _ { i } ^ { 2 } = z _ { i } \in \mathbf { R } } \left(-z_{i}+\int_{[\theta, 1]} \frac{1}{\alpha_{i}} E\left[\left(z_{i}^{1}-X_{i}^{1}\right)^{+}\right.\right.\right.\right. \\
& \left.\left.\left.\left.+\left(z_{i}^{2}-X_{i}^{2}\right)^{+}\right] \mu_{i}\left(d \alpha_{i}\right)\right)\right]_{i=1}^{m}+\mathbf{R}_{+}^{m}\right) \times\{0\}^{d-m} \\
& =\left(\left[\inf _{z_{i}^{1} \in \mathbf{R}}\left(-z_{i}^{1}+\int_{[\theta, 1]} \frac{1}{\alpha_{i}} E\left[\left(z_{i}^{1}-X_{i}^{1}\right)^{+}\right] \mu_{i}\left(d \alpha_{i}\right)\right)\right]_{i=1}^{m}\right. \\
& +\mathbf{R}_{+}^{m}+\left[\inf _{z_{i}^{2} \in \mathbf{R}}\left(-z_{i}^{2}+\int_{[\theta, 1]} \frac{1}{\alpha_{i}} E\left[\left(z_{i}^{2}-X_{i}^{2}\right)^{+}\right] \mu_{i}\left(d \alpha_{i}\right)\right)\right]_{i=1}^{m} \\
& \left.+\mathbf{R}_{+}^{m}\right) \times\{0\}^{d-m} \\
& =W \operatorname{VaR}_{\mu}\left(\mathbf{X}^{1}\right)+W \operatorname{VaR}_{\mu}\left(\mathbf{X}^{2}\right) . \\
& \text { (c) For } u=\left(u_{1}, \cdots, u_{m}\right) \in \mathbf{R}^{m} \text {, }
\end{aligned}
$$

$W \operatorname{VaR}_{\mu}(\mathbf{X}+\bar{u})=$

$$
\begin{aligned}
\left(\left[\operatorname { i n f } _ { z _ { i } \in \mathbf { R } } \left(-z_{i}\right.\right.\right. & \left.\left.+\int_{[\theta, 1]} \frac{1}{\alpha_{i}} E\left[\left(z_{i}-\bar{u}_{i}-X_{i}\right)^{+}\right] \mu_{i}\left(d \alpha_{i}\right)\right)\right]_{i=1}^{m} \\
& \left.+\mathbf{R}_{+}^{m}\right) \times\{0\}^{d-m} \\
= & \left(\left[\inf _{z_{i} \in \mathbf{R}}\left(-\left(z_{i}-\bar{u}_{i}\right)+\int_{[\theta, 1]} \frac{1}{\alpha_{i}} E\left[\left(z_{i}-\bar{u}_{i}-X_{i}\right)^{+}\right] \mu_{i}\left(d \alpha_{i}\right)-\bar{u}_{i}\right)\right]_{i=1}^{m}\right. \\
& \left.+\mathbf{R}_{+}^{m}\right) \times\{0\}^{d-m} \\
= & W \operatorname{VaR}_{\mu}(\mathbf{X})-\bar{u} .
\end{aligned}
$$

(d) Given $\mathbf{X}^{1}=\left(X_{1}^{1}, \cdots, X_{d}^{1}\right), \mathbf{X}^{2}=\left(X_{1}^{2}, \cdots, X_{d}^{2}\right) \in L_{d}^{0}$ with $\mathbf{X}^{2}-\mathbf{X}^{1} \in\left(L_{d}^{0}\right)_{+}$, we have $\left(z_{i}-X_{i}^{2}\right)^{+} \leq\left(z_{i}-X_{i}^{1}\right)^{+}$for each $z_{i} \in \mathbf{R}, 1 \leq i \leq d$. Hence,

$$
\begin{aligned}
& \inf _{z_{i} \in \mathbf{R}}\left(-z_{i}+\int_{[\theta, 1]} \frac{1}{\alpha_{i}} E\left[\left(z_{i}-X_{i}^{2}\right)^{+}\right] \mu_{i}\left(d \alpha_{i}\right)\right) \\
& \quad \leq \inf _{z_{i} \in \mathbf{R}}\left(-z_{i}+\int_{[\theta, 1]} \frac{1}{\alpha_{i}} E\left[\left(z_{i}-X_{i}^{1}\right)^{+}\right] \mu_{i}\left(d \alpha_{i}\right)\right) .
\end{aligned}
$$

Therefore,

$$
\begin{aligned}
{\left[\inf _{z_{i} \in \mathbf{R}}(\right.} & \left.\left.-z_{i}+\int_{[\theta, 1]} \frac{1}{\alpha_{i}} E\left[\left(z_{i}-X_{i}^{2}\right)^{+}\right] \mu_{i}\left(d \alpha_{i}\right)\right)\right]_{i=1}^{m} \\
& \leq\left[\inf _{z_{i} \in \mathbf{R}}\left(-z_{i}+\int_{[\theta, 1]} \frac{1}{\alpha_{i}} E\left[\left(z_{i}-X_{i}^{1}\right)^{+}\right] \mu_{i}\left(d \alpha_{i}\right)\right)\right]_{i=1}^{m} .
\end{aligned}
$$


Consequently,

$$
\begin{gathered}
\left(\left[\inf _{z_{i} \in \mathbf{R}}\left(-z_{i}+\int_{[\theta, 1]} \frac{1}{\alpha_{i}} E\left[\left(z_{i}-X_{i}^{2}\right)^{+}\right] \mu_{i}\left(d \alpha_{i}\right)\right)\right]_{i=1}^{m}\right. \\
\left.+\mathbf{R}_{+}^{m}\right) \times\{0\}^{d-m} \\
\supseteq\left(\left[\inf _{z_{i} \in \mathbf{R}}\left(-z_{i}+\int_{[\theta, 1]} \frac{1}{\alpha_{i}} E\left[\left(z_{i}-X_{i}^{1}\right)^{+}\right] \mu_{i}\left(d \alpha_{i}\right)\right)\right]_{i=1}^{m}\right. \\
\left.+\mathbf{R}_{+}^{m}\right) \times\{0\}^{d-m}
\end{gathered}
$$

which implies that $W \operatorname{VaR}_{\mu}\left(\mathbf{X}^{2}\right) \supseteq W \operatorname{VaR}_{\mu}\left(\mathbf{X}^{1}\right)$.

(e) It is not difficult to verify that $W V^{2} R_{\mu}(\mathbf{X})+\mathbf{M}_{+}=$ $W \operatorname{VaR}_{\mu}(\mathbf{X})$ and that $W \operatorname{VaR}_{\mu}(0)$ is a convex cone.

\subsection{The Market Extension}

The weighted value at risk from Definition 2.1 does not take into account the investment preferences of investors. Therefore, we define its market extension by replacing $\left(L_{d}^{0}\right)_{+}$with a general closed convex cone $K$ containing $\left(L_{d}^{0}\right)_{+}$(see [7] or [8] for further motivation).

Definition 2.2 Let $\widetilde{K}$ be a closed convex cone that contains $\left(L_{d}^{1}\right)_{+}$ and $K$ be a closed convex cone that contains $\left(L_{d}^{0}\right)_{+}$. The extended version of the set-valued weighted value at risk is defined as

$$
\begin{aligned}
\operatorname{WVaR}_{\mu}^{e x t}(\mathbf{X}) & :=\left\{\int_{[\theta, 1]^{d}} \operatorname{diag}(\alpha)^{-1} E[\mathbf{Z}] \mu(d \alpha)-z ;\right. \\
\mathbf{Z} & \left.\in \widetilde{K}, \mathbf{X}+\mathbf{Z}-z \in K, z \in \mathbf{R}^{d}\right\} \cap \mathbf{M} .
\end{aligned}
$$

In the proof of Proposition 2.1, through the same argument, we present the following proposition, which provides another equivalent representation of $W V_{a} R_{\mu}^{e x t}(\cdot)$.

Proposition 2.3 Let $\mathbf{M}=\mathbf{R}^{m} \times\{0\}^{d-m}$. WVaR $R_{\mu}^{\text {ext }}$ has the following equivalent representation:

$$
\begin{aligned}
\operatorname{WVaR}_{\mu}^{e x t}(\mathbf{X})=( & \left(\inf _{z_{i} \in \mathbf{R}}\left(\int_{[\theta, 1]} \frac{1}{\alpha_{i}} E\left[\left(-X_{i}+z_{i}\right)^{+}\right] \mu_{i}\left(d \alpha_{i}\right)-z_{i}\right)\right]_{i=1}^{m} \\
& +C) \times\{0\}^{d-m}
\end{aligned}
$$

where $\mathrm{C}$ is a closed convex cone that contains $\mathbf{R}_{+}^{d}$.

The next proposition will show that when $\mathbf{M}=$ $\mathbf{R}^{m} \times\{0\}^{d-m}, W V a R_{\mu}^{e x t}$ is exactly a set-valued coherent risk measure in the sense of Jouini et al. [7].

Proposition 2.4 Let $\mathbf{M}=\mathbf{R}^{m} \times\{0\}^{d-m}$. Then, the function $\mathbf{X} \longrightarrow W \operatorname{VaR}_{\mu}^{\text {ext }}(\mathbf{X})$ satisfies the following properties:

(a) Positive Homogeneity: for each $\mathbf{X} \in L_{d}^{0}$ and each $s>0$, $W \operatorname{VaR}_{\mu}^{\text {ext }}(s \mathbf{X})=s W \operatorname{VaR}_{\mu}^{\text {ext }}(\mathbf{X})$. (b) Subadditivity: for each $\mathbf{X}^{1}, \mathbf{X}^{2} \in L_{d}^{0}, W V_{a} R_{\mu}^{\text {ext }}\left(\mathbf{X}^{1}+\mathbf{X}^{2}\right) \supseteq$ $W \operatorname{VaR}_{\mu}^{\text {ext }}\left(\mathbf{X}^{1}\right)+W \operatorname{VaR}_{\mu}^{\text {ext }}\left(\mathbf{X}^{2}\right)$.

(c) M-translation invariance: for each $\mathbf{X} \in L_{d}^{0}$ and each $u \in \mathbf{R}^{m}$, $W \operatorname{VaR}_{\mu}^{\text {ext }}(\mathbf{X}+\bar{u})=W \operatorname{VaR}_{\mu}^{\text {ext }}(\mathbf{X})-\bar{u}$, where $\bar{u}=u \times\{0\}^{d-m}$.

(d) Monotonicity with respect to $K$ : for any $\mathbf{X}^{1}, \mathbf{X}^{2} \in K$ and $\mathbf{X}^{2} \succeq_{K} \mathbf{X}^{1}$, which means that $\mathbf{X}^{2}-\mathbf{X}^{1} \in K$, we have $W \operatorname{VaR}_{\mu}^{\text {ext }}\left(\mathbf{X}^{2}\right) \supseteq W \operatorname{VaR}_{\mu}^{\text {ext }}\left(\mathbf{X}^{1}\right)$.

(e) For each $\mathbf{X} \in L_{d}^{0}$, the set $W V_{a} R_{\mu}^{e x t}(\mathbf{X}) \subset \mathbf{M}$ is convex and satisfies that $W \operatorname{VaR}_{\mu}^{\text {ext }}(\mathbf{X})+C_{M}=W \operatorname{VaR}_{\mu}^{\text {ext }}(\mathbf{X})$, where $C_{M}:=C \bigcap M$ and $C$ is as in Proposition 2.3. In particular, $W \operatorname{VaR}_{\mu}^{\text {ext }}(\mathbf{0})$ is a convex cone that satisfies $C_{M} \subseteq W W R_{\mu}^{e x t}(\mathbf{0})$ and $W \operatorname{VaR}_{\mu}^{\text {ext }}(\mathbf{0}) \bigcap-\mathbf{C}_{\mathbf{M}}=\{\mathbf{0}\}$.

Proof: (a) For $\mathbf{X} \in L_{d}^{0}$ and $s>0$, we have

$$
\begin{aligned}
& \operatorname{WVaR}_{\mu}^{e x t}(s \mathbf{X})=\left\{\int_{[\theta, 1]^{d}} \operatorname{diag}(\alpha)^{-1} E[\mathbf{Z}] \mu(d \alpha)-z ; \mathbf{Z} \in \widetilde{K}, s \mathbf{X}\right. \\
&\left.+\mathbf{Z}-z \in K, z \in \mathbf{R}^{d}\right\} \cap \mathbf{M} \\
&=\left\{\int_{[\theta, 1]^{d}} \operatorname{diag}(\alpha)^{-1} E[\mathbf{Z}] \mu(d \alpha)-z ;\right. \\
&\left.\frac{\mathbf{Z}}{s} \in \widetilde{K}, s\left(\mathbf{X}+\frac{\mathbf{Z}}{s}-\frac{z}{s}\right) \in K, \frac{z}{s} \in \mathbf{R}^{d}\right\} \cap \mathbf{M} \\
&=\left\{s\left(\int_{[\theta, 1]^{d}} \operatorname{diag}(\alpha)^{-1} E\left[\frac{\mathbf{Z}}{s}\right] \mu(d \alpha)-\frac{z}{s}\right) ;\right. \\
&\left.\frac{\mathbf{Z}}{s} \in \widetilde{K}, \mathbf{X}+\frac{\mathbf{Z}}{s}-\frac{z}{s} \in K, \frac{z}{s} \in \mathbf{R}^{d}\right\} \cap \mathbf{M} \\
&= s W \operatorname{VaR}_{\mu}^{e x t}(\mathbf{X}) .
\end{aligned}
$$

(b) For $\mathbf{X}^{1}, \mathbf{X}^{2} \in L_{d}^{0}$,

$$
\begin{aligned}
& W V_{a} R_{\mu}^{e x t}\left(\mathbf{X}^{1}\right)+W \operatorname{VaR}_{\mu}^{e x t}\left(\mathbf{X}^{2}\right) \\
&=\left\{\int_{[\theta, 1]^{d}} \operatorname{diag}(\alpha)^{-1} E\left[\mathbf{Z}^{1}\right] \mu(d \alpha)-z^{1}\right. \\
&+ \int_{[\theta, 1]} \operatorname{diag}(\alpha)^{-1} E\left[\mathbf{Z}^{2}\right] \mu(d \alpha)-z^{2} ; \mathbf{Z}^{1}, \mathbf{Z}^{2} \in \widetilde{K}, \mathbf{X}^{1} \\
&\left.+\mathbf{Z}^{1}-z^{1} \in K, \mathbf{X}^{2}+\mathbf{Z}^{2}-z^{2} \in K, z^{1}, z^{2} \in \mathbf{R}^{d}\right\} \cap \mathbf{M} \\
& \subseteq\left\{\int_{[\theta, 1]^{d}} \operatorname{diag}(\alpha)^{-1} E\left[\mathbf{Z}^{1}+\mathbf{Z}^{2}\right] \mu(d \alpha)-\left(z^{1}+z^{2}\right) ;\right. \\
& \mathbf{Z}^{1}+\mathbf{Z}^{2} \in \widetilde{K}, \mathbf{X}^{1}+\mathbf{X}^{2}+\mathbf{Z}^{1}+\mathbf{Z}^{2}-\left(z^{1}+z^{2}\right) \in K, z^{1} \\
&\left.+z^{2} \in \mathbf{R}^{d}\right\} \cap \mathbf{M} \\
&=\left\{\int_{[\theta, 1]^{d}} \operatorname{diag}(\alpha)^{-1} E[\mathbf{Z}] \mu(d \alpha)-z ; \mathbf{Z} \in \widetilde{K}, \mathbf{X}^{1}+\mathbf{X}^{2}+\mathbf{Z}\right. \\
&\left.-z \in K, z \in \mathbf{R}^{d}\right\} \cap \mathbf{M} \\
&= W \operatorname{VaR}_{\mu}^{e x t}\left(\mathbf{X}^{1}+\mathbf{X}^{2}\right) .
\end{aligned}
$$


(c) It is straightforward.

(d) $W V_{a} R_{\mu}^{\text {ext }}$ is $\mathrm{K}$-monotone because for $\mathbf{Y} \in K$, we have $\mathbf{Y}+K \subseteq K$, and therefore, $\operatorname{WVaR}_{\mu}^{\text {ext }}(\mathbf{X}-\mathbf{Y})=$ $\left\{\int_{[\theta, 1]^{d}} \operatorname{diag}(\alpha)^{-1} E[\mathbf{Z}] \mu(d \alpha)-z ; \mathbf{Z} \in \widetilde{K}, \mathbf{X}+\mathbf{Z}-z \in \mathbf{Y}+K, z\right.$ $\left.\in \mathbf{R}^{d}\right\} \cap \mathbf{M} \subseteq W \operatorname{VaR}_{\mu}^{e x t}(\mathbf{X})$.

(e) It is straightforward. Proposition 2.4 is proved.

\section{EXAMPLES}

In this part, we give two examples of computing $W V a R_{\mu}$. In the rest of the paper, we will consider a finite financial market, that is, we assume that $(\Omega, \mathscr{F}, P)$ is a finite probability space. Namely, let $|\Omega|=N, \mathscr{F}=2^{\Omega}, P=\left(p_{1}, p_{2}, \ldots, p_{N}\right)$ with $\sum_{n=1}^{N} p_{n}=1$ and $P\left(\left\{\omega_{n}\right\}\right)=p_{n}, n=1,2, \ldots, N$. Here, $\mathrm{N}$ is a strictly positive number, and the probability measure $P$ is given by $N$.

The first example is motivated by Hamel et al. [11] (Example 3.1).

Example 3.1 Suppose that the elements of a portfolio are $d=2$ and $\mathbf{M}=\mathbf{R}^{2}$ (hence all the initial portfolios are eligible). In a binary model with $N=2$ and $P=(0.4,0.6)$, the potential income is given by

$$
\mathbf{X}\left(\omega_{1}\right)=(12,-20)^{T}, \quad \mathbf{X}\left(\omega_{2}\right)=(4,-6)^{T} .
$$

We set the benchmark level $\theta=0.01$ and let $\mu_{1}=\mu_{2}:=v$. If $v$ is set to be uniformly distributed on $[\theta, 1]$, that is, for Borel measurable set $A \subset[\theta, 1]$,

$$
v(A):=\int_{A} f(x) d x,
$$

where $f(x)=\frac{1}{1-\theta}$ for $\theta \leq x \leq 1$. By a simple calculation, we have that

$$
\operatorname{WVaR}_{\mu}(\mathbf{X})=(-4,20)^{T}+\mathbf{R}_{+}^{2} .
$$

If we let $v$ be a (discrete) probability law with $v(\{0.01\})=$ $v(\{0.02\})=0.5$, then calculation shows that

$$
W \operatorname{VaR}_{\mu}(\mathbf{X})=(-4,20)^{T}+\mathbf{R}_{+}^{2}
$$

again. For the first and second assets, the margins that the manager/regulator needs for compensating the risk are at least 4 units and -20 units, respectively.

In the above example, the value of $W V a R_{\mu}(\mathbf{X})$ is equal to that of $A V @ R_{\alpha}^{r e g}(\mathbf{X})$, the set-valued regulator average value at risk (see [11], Definition 2.1 and Example 3.1), where $\alpha=(0.01,0.02)^{T}$. The next example will show that the values of $W V R_{\mu}(\mathbf{X})$ and $A V @ R_{\alpha}^{r e g}(\mathbf{X})$ are not necessarily the same and that $W V a R_{\mu}(\mathbf{X})$ is better suited to a market featuring extreme events than is $A V @ R_{\alpha}^{r e g}(\mathbf{X})$.

Example 3.2 Let all the input parameters and the potential incomes of $\mathbf{X}$ be as in Example 3.1 except for the probability law
$P$ and the probability measure $\mu$. Here, we set $P=(0.99,0.01)$. If $v$ is set to be uniformly distributed on $[\theta, 1]$, then

$$
W_{\operatorname{VaR}}(\mathbf{X})=(-11.628,20)^{T}+\mathbf{R}_{+}^{2} .
$$

If $v$ is again a (discrete) probability law with $v(\{0.01\})=$ $v(\{0.02\})=0.5$, then,

$$
W_{\operatorname{VaR}}(\mathbf{X})=(-6,20)^{T}+\mathbf{R}_{+}^{2} .
$$

In contrast to the above example, the probability measure $\mu$ concerning the confidence levels does affect the risk measure because the minimal margin to cancel the risk for a manager/regulator covers the worst case only for the second asset, which is -20 units.

On the other hand,

$$
A V @ R_{\alpha}^{r e g}(\mathbf{X})=(-4,20)^{T}+\mathbf{R}_{+}^{2},
$$

where $\alpha=(0.01,0.02)^{T}$, which is the same as in Example 3.1.

From the above two examples, we observe that when all the input parameters remain the same except for the change in the (binary) probability law $P$ from $(0.4,0.6)$ to $(0.99,0.01)$, the minimal risk-compensating portfolio of $W V a R_{\mu}(\mathbf{X})$ changes from $(-4,20)$ to $(-11.628,20)$ and $(-6,20)$, respectively, whereas the minimal risk-compensating portfolio of $A V @ R_{\alpha}^{r e g}(\mathbf{X})$ remains unchanged, which is $(-4,20)$. Thus, we conclude that $W V a R_{\mu}(\mathbf{X})$ can reflect the change in the market, that is, the change in the (binary) probability $P$, whereas $A V @ R_{\alpha}^{r e g}(\mathbf{X})$ cannot. In the case of $P=(0.99,0.01)$, the event with probability 0.01 could be regarded as an extreme event compared with the other event with probability 0.99 . Therefore, we conclude that $W V a R_{\mu}$ is better suited to a market featuring extreme events than is $A V @ R_{\alpha}^{r e g}$.

\section{CONCLUSIONS}

In this paper, we proposed two new classes of set-valued coherent risk measures: the "regulator" version and "market" version. Their essential properties are discussed, and equivalent representations are given. Moreover, the coherency of the setvalued weighted value at risk is characterized. These newly introduced set-valued risk measures complement the study of set-valued risk measures. Examples are also presented that show that set-valued weighted value at risk is better suited to a market featuring extreme events than is $A V @ R_{\alpha}^{r e g}$.

\section{DATA AVAILABILITY STATEMENT}

The original contributions presented in the study are included in the article/supplementary materials, further inquiries can be directed to the corresponding author/s.

\section{AUTHOR CONTRIBUTIONS}

YD, YH, and YF: conceptualization, formal analysis, writingoriginal draft preparation, writing-review and editing, and funding acquisition. All authors contributed to the article and approved the submitted version. 


\section{FUNDING}

This work was supported by the National Key Research and Development Program of China (Grant No. 2018YFB0204403)

\section{REFERENCES}

1. Cherny AS. Weighted V@R and its properties. Finance Stochast. (2006) 10:367-93. doi: 10.1007/s00780-006-0009-1

2. Artzner P, Delbaen F, Eber JM, Heath D. Coherent measures of risk. Math Finance. (1999) 9:203-28. doi: 10.1111/1467-9965.00068

3. Föllmer H, Schied A. Stochastic Finance: An Introduction in Discrete Time. 3rd Edn. Berlin: Walter de Gruyter \& Co (2011).

4. Kusuoka S. On law invariant coherent risk measures. Adv Math Econ. (2001) 3:83-95. doi: 10.1007/978-4-431-67891-5_4

5. Acerbi C. Spectral meausure of risk: a coherent representation of subjective risk aversion. J Bank Finance. (2002) 26:1505-18. doi: 10.1016/S0378-4266(02)00281-9

6. Acerbi C. Coherent representations of subjective risk aversion. In: Szegö G, editor. Risk Measure for the 21st Century. New York, NY: Wiley (2004) p. 147-207.

7. Jouini W, Meddeb M, Touzi N. Vector-valued coheret risk measures. Finance Stochast. (2004) 8:531-52. doi: 10.1007/s00780-004-0127-6

8. Hamel AH. A duality theory for set-valued functions I. Set-Valued Anal. (2009) 17:153-82. doi: 10.1007/s11228-009-0109-0

9. Hamel AH, Heyde F. Duality for set-valued measures of risk. SIAM J Financ Math. (2010) 1:66-95. doi: 10.1137/080743494

10. Hamel AH, Heyde F, Rudloff B. Set-valued risk measures for conical market models. Math Financ Econ. (2011) 5:1-28. doi: 10.1007/s11579-011-0047-0

11. Hamel AH, Rudloff B, Yankova M. Set-valued average value at risk and its computation. Math Financ Econ. (2013) 7:229-46. doi: 10.1007/s11579-013-0094-9

12. Ararat C, Hamel AH, Rudloff B. Set-valued shortfall and divergence risk measures[J]. Int $J$ Theor Appl Finance. (2017) 20:1750026. doi: 10.1142/S0219024917500261

13. Feinstein Z, Rudloff B. Multi-portfolio time consistency for set-valued convex and coherent risk measure. Finance Stoch. (2015) 19:67-107. doi: 10.1007/s00780-014-0247-6

14. Delbaen F. Coherent Risk Measures on General Probability Spaces (Advances in Finance and Stoshatics). Berlin; Heidelberg; New York, NY: Springer (2002).

15. Hamel AH. Translative Sets and Functions and Their Applications to Risk Measure Theory and Nonlinear Separation (2006). Available online at: https:// fam.tuwien.ac.at/events/abstracts/20060606.pdf.

16. Ararat C. On set-valued functionals: multivariate risk measures and Aumann integrals[J] (Dissertations \& Theses - Gradworks). Princeton University, Princeton, NJ, United States (2015).

17. Artzner P, Delbaen F, Eber JM, Heath D. Thinking coherently. Risk. (1997) 10:68-71. and Shenzhen Grade E Scientific and Engineering Calculation Key Laboratory under Grant No. ZDSYS201703031711426. It was also supported by the National Natural Science Foundation of China (No. 1137 1284).

18. Chen J, Wang C, Zhang X-A, Ha M. A further discussion of structural risk minimization principle on set-valued probability space[C]. In: Proceedings of International Conference on Machine Learning and Cybernetics, ICMLC. Qingdao: IEEE (2010).

19. Yu F, Yichuan D, Jia-Bao L. Set-valued haezendonck-goovaerts risk measure and its properties[J]. Discrete Dyn Nat Soc. (2017) 2017:1-7. doi: $10.1155 / 2017 / 5320908$

20. Deprez O, Gerber HU. On convex principles of premium calculation. Insurance. (1985) 4:179-89. doi: 10.1016/0167-6687(85)90014-9

21. Frittelli M, Rosazza Gianin E. Putting order in risk measures. J Bank Finance. (2002) 26:1473-86. doi: 10.1016/S0378-4266(02)00270-4

22. Artzner P, Delbaen F, Koch-Medina P. Risk measures and efficient use of capital. Astin Bull. (2009) 39:101-16. doi: 10.2143/AST.39.1.2038058

23. Rüschendorf L. Mathematical Risk Analysis[M]. Berlin; Heidelberg: Springer (2013).

24. Föllmer H, Schied A. Convex measures of risk and trading constraints. Finance Stochast. (2002) 6:429-47. doi: 10.1007/s007800200072

25. Cascos I, Molchanov I. Multivariate risks and depth-trimmed regions. Finance Stochast. (2007) 11:373-97. doi: 10.1007/s00780-007-0043-7

26. Burgert C, Rüschendorf L. Consistent risk measures for portfolio vectors. Insurance. (2006) 38:289-97. doi: 10.1016/j.insmatheco.2005.08.008

27. Embrechts P, Puccetti G. Bounds for functions of multivariate risks. J Multi Var Anal. (2006) 97:526-47. doi: 10.1016/j.jmva.2005.04.001

28. Basel Committee. Amendment to the Capital Accord to Incorporate Market Risks. Basle Committee on Banking Supervision (1996).

29. Basel Committee. International Convergence of Capital Measurement and Capital Standards: A Revised Framework (Comprehensive Version). Basle Committee on Banking Supervision (2006).

30. Basel Committee. Guidelines for Computing Capital for Incremental Risk in the Traing Book. Basel Committee on Banking Supervision (2009).

31. Basel Committee. Revision to the Basel II Market Risk Framework. Basel Committee on Banking Supervisiom (2011).

Conflict of Interest: The authors declare that the research was conducted in the absence of any commercial or financial relationships that could be construed as a potential conflict of interest.

Copyright (c) 2020 Dong, Hu and Feng. This is an open-access article distributed under the terms of the Creative Commons Attribution License (CC BY). The use, distribution or reproduction in other forums is permitted, provided the original author(s) and the copyright owner(s) are credited and that the original publication in this journal is cited, in accordance with accepted academic practice. No use, distribution or reproduction is permitted which does not comply with these terms. 